\title{
Entanglement of Quantum Evolutions
}

\author{
Paolo Zanardi \\ Institute for Scientific Interchange (ISI) Foundation, Viale Settimio Severo 65, I-10133 Torino, Italy \\ Istituto Nazionale per la Fisica della Materia (INFM)
}

(October 30, 2018)

\begin{abstract}
The notion of entanglement can be naturally extended from quantum-states to the level of general quantum evolutions. This is achieved by considering multi-partite unitary transformations as elements of a multi-partite Hilbert space and then extended to general quantum operations. We show some connection between this entanglement and the entangling capabilities of the quantum evolution.
\end{abstract}

PACS numbers: 03.67.Lx, 03.65.Fd

It is theoretically rewarding to describe the physical world in terms of subsystems. It follows that it is a fundamental kinematical requirement to be able to describe the state-space of a composite system in terms of the simpler state-spaces associated with its parts. In quantum theory a basic axioms states that: The state space associated with a bi-partite quantum system made out of two subsystems $S_{1}$ and $S_{2}$ is given by the tensor product of the state-spaces associated with the $S_{i}$ 's [1].

This fact has been in the recent years shown to be at the basis of many of the novel quantum capabilities in information processing and computational power [2]. Roughly speaking this is due to the fact that, on the one hand the tensor product rule gives rise to exponentially large state-spaces in which information can be encoded. On the other hand the very existence of entangled states i.e., not product states with their characteristic correlations, amounts to a new kind of uniquely quantum computational resource. A lot of efforts have been accordingly made aimed to the understanding of the entanglement of quantum states [3].

Clearly the generation of such entangled states is a subject that has interest on its own. Therefore more recently attention started to be devoted to the entangling capabilities of quantum evolutions. Both from the point of view of average entangling power [A] of a general $d_{1} \times d_{2}$ unitary transformation and from the point view of designing optimal strategies for entanglement production [5].

In this paper we address the related issue of entanglement of quantum evolutions. This notion arises in a very simple way once one recalls that unitary operators realizing the (closed) quantum dynamics of a multi-partite system belong to a multi-partite state-space as well, the so-called Hilbert-Schmidt space. One is therefore naturally led to lift all the notions developed so-far for quantum state entanglement to the operatorial realm. The point is to see whether, beyond their obvious mathematical meaning, such concepts at the operator level may provide some novel physical insight.

In the following we shall move the first steps of this programme by introducing an entanglement measure $E$ over the operator space over a bi-partite $d \times d$ state-space. A quantum protocol that provides $E$ with a simple operational interpretation will be described. We shall study $E$ analytically obtaining explict results for abitrary dimension $d$. Moreover we shall show how this operator entanglement can be extended to general quantum evolutions; such an extension will allow us to reinterpret in a quite natural way the mapping between quantum operations and quantum states very recently discussed by Cirac et al [6]. Finally we shall make a connection between operator entanglement and the entangling power for bi-partite unitary evolutions introduced in 绝.

Let us begin by recalling some basic definitions of operator algebras. Let $\mathcal{H}$ be a $d$-dimensional Hilbert space. The algebra of linear operators over $\mathcal{H}$ has on its own a natural structure of $d^{2}$-dimensional Hilbert space. The scalar product between two operators $A$ and $B$ is provided by the Hilbert-Schmidt product: $\langle A, B\rangle:=$ $\operatorname{tr}\left(A^{\dagger} B\right),\|A\|_{H S}:=\sqrt{\langle A, A\rangle}$. When the operator algebra is thought of as endowed with such structure it will be denoted by $\mathcal{H}_{H S}$ and accordingly the ket notation will be used for operators. From the basic fact that the space of linear operators over a tensor product is given by the tensor product of linear operators. it follows

$$
\left(\mathcal{H}^{\otimes 2}\right)_{H S} \cong \mathcal{H}_{H S}^{\otimes 2} .
$$

The space of operators associated with a bi-partite quantum system is on itself a bi-partite quantum state-space. From this remark it stems that all the notions and tools developed so far for the study of entanglement of quantum states lift to the operator level in a straightforward way. In particular one can consider unitaries $U$ in $\mathcal{H}_{H S}^{\otimes 2}$ as representing all the possible evolutions of a (closed) bi-partite quantum system with state-space $\mathcal{H}^{\otimes 2}$.

To address the issue of operator entanglement in a quantitative fashion it is useful to recall that the HilbertSchmidt space $\mathcal{H}_{H S}$ is isomorphic to $\mathcal{H}^{\otimes 2}$ not just algebraically - in that they have the same dimension but also as Hilbert spaces. Indeed there exists a natural Hilbert-space isomorphism $\Psi$ between $\mathcal{H}_{H S}$ and $\mathcal{H}^{\otimes 2}$ given by 


$$
\Psi: X \mapsto(X \otimes \mathbb{1})\left|\Phi^{+}\right\rangle, \quad\left|\Phi^{+}\right\rangle:=\sum_{\alpha=1}^{d}|\alpha\rangle^{\otimes 2}
$$

where $\{|\alpha\rangle\}_{\alpha=1}^{d}$ is an orthonormal basis of $\mathcal{H}$. The relation (2) defines indeed a unitary transformation: $\langle\Psi(X), \Psi(Y)\rangle=\left\langle\Phi^{+}\left|\left(X^{\dagger} Y\right) \otimes \mathbb{1}\right| \Phi^{+}\right\rangle=$ $\sum_{\alpha=1}^{d}\left\langle\alpha\left|X^{\dagger} Y\right| \alpha\right\rangle=\operatorname{tr}\left(X^{\dagger} Y\right)=<X, Y>$. Even though $\Psi$ does not preserve the algebraic structure of $\mathcal{H}_{H S}$ it has the property of mapping the group $\mathcal{U}(\mathcal{H})$ onto the manifold of maximally entangled states of $\mathcal{H}^{\otimes 2}$.

Moving to the bi-partite case by tensoring one obtains a unitary map $\Psi$ (strictly speaking it would be $\Psi^{\otimes 2}$ ) between $\mathcal{H}_{H S}^{\otimes 2}$ and $\mathcal{H}^{\otimes 2} \otimes \mathcal{H}^{\otimes 2} \cong \mathcal{H}^{\otimes 4}$ that associates with the operator $X$ the vector

$$
|\Psi(X)\rangle:=\left(X_{13} \otimes \mathbb{1}_{24}\right)\left|\Phi^{+}\right\rangle^{\otimes 2}, \quad\left(X \in \mathcal{H}_{H S}^{\otimes 2}\right)
$$

where the indexes denotes the factors in $\mathcal{H}^{\otimes 4}$ on which the corresponding operators have a non-trivial action.

The extension of $\Psi$ to general quantum operations i.e.,completely positive (CP) maps is performed by observing that, in view of the operator sum representation theorem [7], one has that any CP-map $T$ acting on quantum states $\rho$, can be written as $T(\rho)=\sum_{i} A_{i} \rho A_{i}^{\dagger},\left(A_{i} \in\right.$ $\left.\mathcal{H}_{H S}\right)$. This allows to associate with $T$ the operator over $\mathcal{H}^{\otimes 4}$ given by

$$
\sum_{i}\left|\Psi\left(A_{i}\right)\right\rangle\left\langle\Psi\left(A_{i}\right)\right|=\left(T_{13} \otimes \mathbb{1}_{24}\right)\left(\left|\Phi^{+}\right\rangle\left\langle\Phi^{+}\right|\right)^{\otimes 2} .
$$

In particular when $T$ is pure i.e., just one $A_{i}$ one gets a pure state consistently with (3). From equation (㺼) it is straightforward to realize that the above mapping is - up to normalization factor - the one-to-one correspondence between quantum operations and quantum states discussed in Ref. [6] by Cirac et al for studying separability properties of quantum evolutions [see their Eq. (4)]. The idea is to extract information about the CP map $T$ from the (unnormalized) state $\Psi(T)$ taking adavantage from the large set of tools developed to the date for studying entanglement of quantum states. In particular in Ref. [6] have been presented protocols based on local operations and classical communications to implement a non-local transformation $U$ by sharing the entangled state $|\Psi(U)\rangle$. It is remarkable that such a powerful correspondence between quantum operations and positive operators simply stems from the natural extension of the basic map (2).

Given an orthonormal basis $\left\{e_{j}\right\}_{j=1}^{d^{2}}$ for $\mathcal{H}_{H S}$ any unitary can be written as $U=\sum_{i, j=1}^{d^{2}} \lambda_{i j} e_{i} \otimes e_{j}$. Since the mapping $\Psi$ associates with $U$ a state with the same $\lambda_{i j}$ 's i.e., $|\Psi(U)\rangle:=\sum_{i, j=1}^{d^{2}} \lambda_{i j}\left|\Psi\left(e_{i}\right)\right\rangle \otimes\left|\Psi\left(e_{j}\right)\right\rangle$, it should be clear that the entanglement properties of $U$ and $|\Psi(U)\rangle$ are the same. Indeed all the entanglement measures $E(U)$ depend just on the singular values $\left\{\lambda_{k}\right\}_{k=1}^{r}$ of the matrix $\lambda=\left(\lambda_{i j}\right)\left(r=d^{2}-\operatorname{dim} \operatorname{Ker} \lambda\right.$ is the rank of $\left.\lambda\right)$.
In particular $U$ and $|\Psi(U)\rangle$ have the same Schmidt decomposition [1]. Once that an entanglement measure $E$ is given it makes therefore sense to define $E(\Psi(U))$ as the entanglement of the quantum evolution $U$. The generalization to an arbitrary $C P$-map $T$ is obtained by the formula $E(T):=E(\Psi(T))$ where now $E$ in the lhs is mixed state-entanglement [8].

We observe that from the fact that entanglement measures for states are not increasing under local operations and classical communications (LOCC) it follows that the entanglement of an operation $T$ does not increase if $T$ is followed by LOCC. This is easily seen as follows. Let $L=\sum_{i} A_{i} \otimes B_{i}$ be a LOOC transformation. From the identity $|\Psi(X Y)\rangle=\left(X_{13} \otimes \mathbb{1}_{24}\right)|\Psi(Y)\rangle$ it follows that $|\Psi(L T)\rangle=\tilde{L}|\Psi(T)\rangle$, where $\tilde{L}:=\sum_{i}\left(A_{i} \otimes \mathbb{1}\right) \otimes\left(B_{i} \otimes \mathbb{1}\right)$. Since this latter map is LOOC for the bi-partite system $\mathcal{H}^{\otimes 2} \otimes \mathcal{H}^{\otimes 2}$ one has $E(|\Psi(L T)\rangle)=E(\tilde{L}|\Psi(T)\rangle) \leq$ $E(|\Psi(T)\rangle)$.

In the remainder of the paper we shall adopt as entanglement measure of the (normalized) $|U\rangle \in \mathcal{H}_{H S}^{\otimes 2}$ the linear entropy of the reduced density matrix

$$
E(U):=1-\operatorname{Tr} \rho_{U}^{2}, \quad \rho_{U}:=\operatorname{Tr}|U\rangle\langle U| .
$$

Even though the information theoretic content of the functional $E$ is less direct than the von Neumann entropy $S\left(\rho_{U}\right)=-\operatorname{Tr}\left(\rho_{U} \ln \rho_{U}\right)$ the linear entropy has the distinct advantage of being a polynomial in $U$. This algebraic simplicity makes possible to find for (5) explicit expressions from which the main features of our operator entanglement measure can be easily derived.

Let $T_{13}$ be the permutation (swap) operator between the first and the third factor of $\mathcal{H}^{\otimes 4}$ and let us denotes by $\hat{T}_{13}$ its adjoint action i.e., $\hat{T}_{13}(X):=T_{13} X T_{13}$. Reasoning as in Ref. [4 it is not difficult to show that one can write

$$
E(U)=1-\frac{1}{d^{4}}<U^{\otimes 2}, \hat{T}_{13}\left(U^{\otimes 2}\right)>.
$$

Notice that the term $d^{4}$ is nothing but the normalization factor of $U^{\otimes 2}\left(\|U\|_{H S}^{2}=\operatorname{tr}\left(U^{\dagger} U\right)=\operatorname{tr} \mathbb{1}=d^{2}\right)$, for a generic -not unitary - $X$ it must be replaced by $\|X\|^{4}$.

Equation (6) allows us to give to our measure of operator entanglement a direct operational meaning. To this aim we introduce the projectors $P_{13}^{ \pm}:=2^{-1}\left(\mathbb{1} \pm T_{13}\right) \otimes \mathbb{1}_{24}$ over the subspaces corresponding to the eigenvalues \pm 1 of $T_{3}$. The operator $\rho_{13}^{+}:=2 P_{13}^{+} /\left[d^{3}(d+1)\right]$ therefore represents the uniform state over the eigenvalue 1 subspace. Eq. (6) can be cast in the form

$$
E(U)=2 N_{d}<U^{\otimes 2} \rho_{13}^{+} U^{\dagger \otimes 2}, P_{13}^{-}>.
$$

From this there follows that (apart from the numerical factor $\left.N_{d}:=(d+1) / d\right), E(U)$ can be viewed - and then in principle measured - as the probability of success of the following protocol in $\mathcal{H}^{\otimes 4}$ 
a) Prepare the state $\rho_{13}^{+}$; b) Let it evolve it by $U^{\otimes 2}$; c) Project on the eigenvalue -1 eigenspace of $T_{13}$.

Now we shall derive the properties of $E: \mathcal{H}_{H S}^{\otimes 2} \mapsto \mathbb{R}$ directly form Eq. (6).

a) First of all let us observe that from the relation $\left[T_{13},\left(U_{1} \otimes U_{2}\right)^{\otimes 2}\right]=0$, it follows that $, \forall U_{1}, U_{2} \in \mathcal{U}(\mathcal{H}$

$$
E\left[\left(U_{1} \otimes U_{2}\right) U\right]=E\left[U\left(U_{1} \otimes U_{2}\right)\right]=E(U) .
$$

This feature is not a peculiar property of the linear entropy; it nothing but the invariance of $E$ under the $l o$ cal unitary transformations of $\mathcal{H}_{H S}^{\otimes 2}$. In other words $E$ is constant along the orbit of unitary elements generated by the $\mathcal{U}(\mathcal{H})^{4}$ - action in $\mathcal{H}_{H S}^{\otimes 2}$ given by $\prod_{i=1}^{4} U_{i} \times U \mapsto$ $\left(U_{1} \otimes U_{2}\right) U\left(U_{3}^{\dagger} \otimes U_{4}^{\dagger}\right)$. These transformations define a $4 d^{2}$-dimensional subgroup of $\mathcal{U}\left(\mathcal{H}_{H S}^{\otimes 2}\right)$, that has the peculiar property of mapping unitaries onto unitaries. The orbit of $U$ will be even referred to as the local equivalence class of $U$.

b) From $d^{2}=\|U\|_{H S}^{2},\left\|\hat{T}_{13}\right\|=1$ and using CauchySchwarz inequality one has $<U^{\otimes 2}, \hat{T}_{13}\left(U^{\otimes 2}\right)>\leq$ $\|U\|_{H S}^{2}\left\|\hat{T}_{13}\left(U^{\otimes 2}\right)\right\|_{H S} \leq\left\|\hat{T}_{13}\right\|\|U\|_{H S}^{4}=d^{4}$. Therefore $E(U) \geq 0$. Notice that $E$ is also invariant under hermitean conjugation: $E(U)=E(U)^{*}=E\left(U^{\dagger}\right)$.

c) From the previous point it follows that

$$
E(U)=0 \Leftrightarrow \hat{T}_{13}\left(U^{\otimes 2}\right)=U^{\otimes 2} \Leftrightarrow\left[T_{13}, U^{\otimes 2}\right]=0,
$$

On the other hand point a) ensures that one can consider, without loss of generality, just the transformations with the form (Schmidt decomposition) $U=\sum_{k=1}^{r} \lambda_{k} e_{k} \otimes$ $e_{k},\left(r \leq d^{2}\right)$. Inserting this expression in the fixed-point equation (9) one finds $k \neq h \Rightarrow \lambda_{k} \lambda_{h}=0$ that in turn implies one must have just one non-vanishing Schmidt coefficient. This means that $U$ is a tensor product i.e., the zero locus of $E$ is the local equivalence class of the identity. Notice that the separability condition (9) applies to all pure operations $T$.

d) Since $E(U)=1-\sum_{k}\left|\lambda_{k}\right|^{4}$ and $\sum_{k}\left|\lambda_{k}\right|^{2}=1$ one recovers the well-known upper bound $E(U) \leq 1-1 / d^{2}$. Such a bound is met by all the elements in the unitary orbit of the swap operator in $\mathcal{H} \otimes \mathcal{H}$. Indeed from Eq. (6) one has: $E(S)=1-d^{-4} \operatorname{tr}\left[(S \otimes S) T_{13}(S \otimes S) T_{13}\right]=$ $1-d^{-4} \operatorname{tr}\left[T_{24} T_{13}\right]=1-1 / d^{2}$. Obviously these maximally entangled transformations are the ones having $d^{2}$ non-vanishing Schmidt coefficients with the same amplitude.

e) The manifold $\mathcal{U}\left(\mathcal{H}^{\otimes 2}\right)$ endowed with the Haar measure $d U$ [12] becomes a probability space over which the operator entanglement (6) defines a random variable. Resorting to group-theoretic arguments it is possible to compute the average of $E$ explicitly [10]:

$$
\overline{E(U)}^{U}:=\int_{\mathcal{U}(\mathcal{H} \otimes 2)} d U E(U)=\frac{d^{2}-1}{d^{2}+1} .
$$

It is interesting to notice that unitary operators have on average higher entanglement than generic operators.
Taking the (uniform) average of (6) over the full unit ball of $\mathcal{H}_{H S}^{\otimes 2}$ one obtains $\overline{E(X)}{ }^{X}=1-<T_{13}|Q| T_{13}>$ in which $Q:=\int_{\|X\|_{H S}=1} d X|X><X|^{\otimes 2}$. Now it is easy to see that $Q \geq 1 / d^{4} \pi$ in that the latter operator is just the restriction to the unitary submanifold of the unit ball in $\mathcal{H}_{H S}$ of the same integral of the former. It follows that $<T_{13}|Q| T_{13}>\geq<T_{13}|\pi| T_{13}>$, which in turn implies the announced inequality.

In order to provide some exemplifications of the measure (6) now we consider a couple of very simple cases

1) Let $\left\{\Pi_{\alpha}\right\}_{\alpha=1}^{r}$ be a set of orthogonal projectors such that $\sum_{\alpha} \Pi_{\alpha}=\mathbb{1}$ and $\left\{U_{\alpha}\right\}_{\alpha=1}^{d}$ is a set of orhogonal unitaries. One can write the controlled unitary operation over $\mathcal{H}^{\otimes 2} U=\sum_{\alpha} \Pi_{\alpha} \otimes U_{\alpha}$. one finds that $E(U)=$ $1-1 / d^{2} \sum_{\alpha}\left|\operatorname{tr} \Pi_{\alpha}\right|^{2}$. Of course the most (least) entangled situation i.e., $E(U)=1-1 / d(E=0)$, corresponds to having all the $\Pi_{\alpha}$ 's one-dimensional $\left(r=1, \Pi_{1}=\mathbb{1}\right)$.

2) Let $\left\{U_{\theta}\right\}_{\theta \in[0,2 \pi)}$ be the one-parameter family of $2 \times 2$ unitary transformations given by $U_{\theta}:=\exp \left[i \theta \sigma_{z}^{\otimes 2}\right]=$ $\cos (\theta) \mathbb{1}^{\otimes 2}+i \sin (\theta) \sigma_{z}^{\otimes 2}$, where $\sigma_{z}=\operatorname{diag}(1,-1)$. It is straightforward to obtain: $E\left(U_{\theta}\right)=2^{-1} \sin ^{2}(2 \theta)$, which clearly displays the separable (maximally entangled) character of the $U_{\theta}$ for $\theta=0, \pi / 2(\theta=\pi / 4)$.

It is worthwhile to mention that the entanglement measure (6) has a simple group-theoretic content in that it is twice the expectation value of the projector $P_{13}^{-}$on the (normalized) state $|U\rangle^{\otimes 2}$. The more this latter state is antisymmetric with respect to the action of the swap $T_{13}$ the more it is entangled. In particular for an unentangled $|U\rangle$ one has that $|U\rangle^{\otimes 2}$ is completely symmetric and one gets the quadratic relation Eq. (9). Interestingly enough this characterization of product states extends to the multi-partite case. Let $\mathcal{H} \cong\left(\mathbb{C}^{d}\right)^{\otimes N}$, and $T_{i, i+N}$ be the swap between the $i$-th and the $i+N$-th factor in $\mathcal{H}^{\otimes 2}$. Since $|\Phi\rangle$ is a product state iff the $N$ single-subsystem reduced density matrices are one-dimensional projectors, one finds that for a $|\Phi\rangle$ to be a product a necessary and sufficient condition is

$$
\left(\mathbb{1}-T_{i, i+N}\right)|\Phi\rangle^{\otimes 2}=0,
$$

where $i=1, \ldots, N-1$. These equatione are just the operator version of the condition given in Ref. [9].

We finally discuss the relation of the entanglement of operator $U$ with its entangling power [四]. It must be stressed that such a relation cannot be trivial. Indeed the more entangled is an operator the more it is nonlocal, but this does not mean that the greater are its entangling capabilities [at least in the sense discussed in [4]. For example the swap operator $S$ maps product states onto product states and therefore has not direct entangling capabilities. On the other hand we have seen that $S$ is maximally entangled. 
In Ref. [- 4 we defined the entangling power $e_{p}(U)$ of a unitary $U$ over $\mathcal{H}^{\otimes 2}$ as the average of the entanglement $E(U|\Psi\rangle)$, where the $|\Psi\rangle$ 's are product states generated according to some given probability distribution $p$. By choosing for $E$ the linear entropy of the reduced density matrix and using an uniform i.e., $U(d) \times U(d)$ invariant $p$ for the $|\psi\rangle$ 's one finds [4] $e_{p}(U)=1-d^{4}<$ $U^{\otimes 2} \rho_{13}^{+} \rho_{24}^{+} U^{\dagger \otimes 2}, T_{13}>$. Comparing this equation with (6) straightforward algebra reveals that

$$
e_{p}(U)=N_{d}^{-2}[E(U)+E(U S)-E(S)]
$$

The $U$-dependent part of the entangling power of the evolution $U \in \mathcal{U}\left(\mathcal{H}^{\otimes 2}\right)$ is proportional to the operator entanglement of $U$ averaged with respect to the multiplicative action of the permutation group $\mathcal{S}_{2}:=\{\mathbb{1}, S\}$. Another way to express Eq. (12) is as the average of $E$ along the $\mathcal{S}_{2}$-orbit of $U$ minus the average entanglement of $\mathcal{S}_{2}$ itself. Notice that the first two terms of Eq. (12) define two independent elements of the ring of polynomial invariants of $U$ 14.

Clearly the simple relation 12) holds just when $E$ is the linear entropy. On the other hand the structure of 12 ) ensures that $e_{p}$ is good entangling power measure whatsoever a good entanglement measure $E$ is chosen. It is therefore tempting to suggest to use a non-decreasing real-valued smooth function of Eq. (12) vanishing at 0 in order to define an entangling power measure for any $E$.

Employing Eq. (12) it is very easy to get the upper bound on the entangling power derived in [4] (Eq. (9) with $\left.d_{1}=d_{2}=d\right)$. Since $E(S)=\max _{U} E(U)=$ $\left(d^{2}-1\right) / d^{2}$ one from Eq. (12) immediately obtains

$$
e_{p}(U) \leq N_{d}^{-2} E(S)=\frac{d-1}{d+1}
$$

Furthermore it is clear that in order for a $U$ to meet such bound i.e, to be optimal in the language of Ref. [4], a unitary $U$ must satisfy the constraints

$$
E(U)=E(U S)=E(S)
$$

Then an optimal transformation $U$ must be maximally entangled, and such that $U S$ is maximally entangled as well. In terms of the $\mathcal{S}_{2}$-action discussed above one can state the optimal transformations are characterized by the fact that the entanglement of $U$ is constant along its $\mathcal{S}_{2}$-orbit and maximal. In view of its simplicity such a statement might be helpful in the search, for optimal unitaries [13]. Notice that property (14) is obviously maximally violated by unitaries belonging to the local equivalence classes of the identity and of the swap.

In this paper we discussed the notion of entanglement of a quantum evolution. This has been done by simply observing that all the notions developed for quantumstate entanglement make sense for quantum evolutions as well. Indeed operators acting on multi-partite quantum state-spaces belong on their own to multi-partite Hilbert spaces. This allows one to introduce entanglement measures $E$ for unitary transformations and then to extend them to general quantum operations. Adopting as $E$ the linear entropy one can obtain analytical expressions for the entanglement of a unitary $U$ and make a simple connection with its entangling power. Here we focused on the bi-partite case but it should be clear that the main idea of lifting the notion of entanglement to the operatorial level extends in a straightforward manner to the multi-partite case [15].

I would like to thank Ch. Zalka for critical discussions and M. Rasetti for a careful reading of the manuscript.

[1] A. Peres, Quantum Theory, Concepts and Methods, Kluwer Academic Publishers, The Netherlands (1995)

[2] For reviews, see D.P. Di Vincenzo and C. Bennet Nature 404, 247 (2000); A. Steane, Rep. Prog. Phys. 61, 117 (1998)

[3] M. Horodecki, P. Horodecki and R. Horodecki, in "Quantum Information - Basic Concepts and Experiments", Eds. G. Alber and M. Weiner, in print (Springer, Berlin, 2000).

[4] P. Zanardi, Ch. Zalka, L. Faoro, Phys. Rev. A 62, 30301 (2000)

[5] W. Dür et al, quant-ph/0006034

[6] I. Cirac et al, Phys. Rev. Lett. 86, 544 (2001)

[7] K. Kraus, States, Effects, and Operations: Fundamental Notions of Quantum Theory, Lecture Notes in Physics, 190, Springer, Berlin (1983)

[8] C.H. Bennett et al., Phys. Rev A 54, 3824 (1998); V. Vedral et al., Phys. Rev. Lett. 78, 2275 (1997).

[9] J. Brylinski, quant-ph/0008031

[10] Eq. (6) can be written as $\overline{E(U)}^{U}=1-d^{-4}<$ $T_{13}|\pi| T_{13}>$ where $\pi:=\int d U|U><U|^{\otimes 2}$, is the projector over the subspace of $\mathcal{H}_{H S}^{\otimes 2} \otimes \mathcal{H}_{H S}^{\otimes 2}$ commuting with all the $U^{\otimes 2}$ 's [11. Such subspace is spanned by $\mathbb{1}$ and $T_{13} T_{24}$ [12]. An orthonormal basis is given by $\left|0>:=\mathbb{1} / d^{2},\right| 1>:=\left(d^{4}-1\right)^{-1 / 2}\left(T_{13} T_{24}-\mathbb{1} / d^{2}\right)$ Using this basis one has $\pi=\sum_{i=0,1}|i><i|$, hence $<T_{13}|\pi| T_{13}>=\sum_{i=1,2}|<i| T_{13}>\left.\right|^{2}$, from which Eq. (10) follows.

[11] P. Zanardi, Phys. Lett. A 258, 77 (1999)

[12] J.F. Cornwell, Group Theory in Physics (Academic, New York, 1984), Vol. I-III

[13] In ref. [4] it has been shown that for $d=2$ maximally entangling unitaries e.g., the c-not, are not optimal.

[14] M. Grassl et al, Phys. Rev. A, 58, 1833 (1998); Yu. Makhlin, quant-ph/0002045

[15] M. Murao et al., Phys. Rev. A 57, R4075 (1998); W. Dür, J. I. Cirac, and R. Tarrach, Phys. Rev. Lett. 83, 3563 (1999); N. Linden, S. Popescu, and A. Sudbery, Phys. Rev. Lett. 83, 243 (1999). 